\title{
EFFECTS OF PREVIOUS UNSUCCESSFUL EXTRACORPOREAL SHOCKWAVE LITHOTRIPSY TREATMENT ON THE PERFORMANCE AND OUTCOME OF PERCUTANEOUS NEPHROLITHOTOMY
}

\author{
${ }_{13}^{\text {rd }}$ Year Mch Resident, Department of Urology, IGIMS, Patna. \\ ${ }^{2} 1^{\text {st }}$ Year Mch Resident, Department of Urology, IGIMS, Patna. \\ ${ }^{33^{r d}}$ Year Mch Resident, Department of Urology, IGIMS, Patna. \\ 4Junior Consultant, MVA Hospital, Patna.
}

Anshul Garg1, Kumar Gaurav Mishra², Pawan Kumar Bharti³, Urvashi Mishra ${ }^{4}$

\section{ABSTRACT}

\section{BACKGROUND}

Majority of renal stones diagnosed today are below $2 \mathrm{~cm}$, perhaps due to easy and early accessibility to x-ray and ultrasonography. The preferred treatment of $<1 \mathrm{~cm}$ stone is extracorporeal shockwave lithotripsy (ESWL), while standard of care for renal stone $>2$ $\mathrm{cm}$ is percutaneous nephrolithotomy (PCNL). The procedure of choice for $1-2 \mathrm{~cm}$ renal stones is still a subject of debate. Controversy exists with regard to optimum management of these stones by percutaneous nephrolithotomy (PCNL) and extracorporeal shockwave lithotripsy (ESWL) with reference to stone clearance, hospital stay and complications. The primary goal while treating renal stones is to achieve complete clearance, while causing minimal morbidity to the patient. Treatment of renal stone depends on stone size and location plus patient related factors including anatomy of pelvicalyceal system. In our study, previous ESWL treatment had no influence on the PCNL stone-free rate, operation time, incidence of postoperative complications and LOS in patients with similar stone burdens. However, bleeding during PCNL was more prevalent in the ESWL-treated patients, so close attention should be paid to bleeding in patients who have been pretreated with ESWL.

The objective of this study is to evaluate the effects of previous unsuccessful extracorporeal shockwave lithotripsy (ESWL) treatment on the performance and outcome of percutaneous nephrolithotomy (PCNL).

\section{MATERIALS AND METHODS}

Of 115 PCNL procedures performed in our institute with similar stone burden and number of accesses was included in the present study. We categorised the study patients into two groups according to whether they underwent ESWL within 1 year prior to PCNL or not. Accordingly, Group 1 comprised of 42 (36.5\%) ESWL-treated patients and Group 2 comprised of 73 (63.4\%) non-ESWLtreated patients.

\section{RESULTS}

There were no statistically significant differences between the groups for length of hospital stay (LOS), nephrostomy tube removal time and the presence of residual stones. When we evaluated the groups for both the preoperative and postoperative haemoglobin $(\mathrm{Hb})$ drop and blood transfusion rate, manifest $\mathrm{Hb}$ declined and more transfusions were required in the ESWL-treated patients (both $\mathrm{P}=0.01$ ).

\section{CONCLUSION}

Many patients who undergo PCNL have undergone ESWL treatment beforehand. The renal side-effects of ESWL are well known, the duration of which varies between studies. In the present study, we found that previous ESWL had an effect on bleeding during the PCNL procedure.

\section{KEYWORDS}

Kidney Stones, ESWL, Percutaneous, Nephrolithotomy, Bleeding Complications.

HOW TO CITE THIS ARTICLE: Garg A, Mishra KG, Bharti PK, et al. Effects of previous unsuccessful extracorporeal shockwave lithotripsy treatment on the performance and outcome of percutaneous nephrolithotomy. J. Evolution Med. Dent. Sci. 2017;6(75): 5408-5412, DOI: $10.14260 /$ Jemds/2017/1172

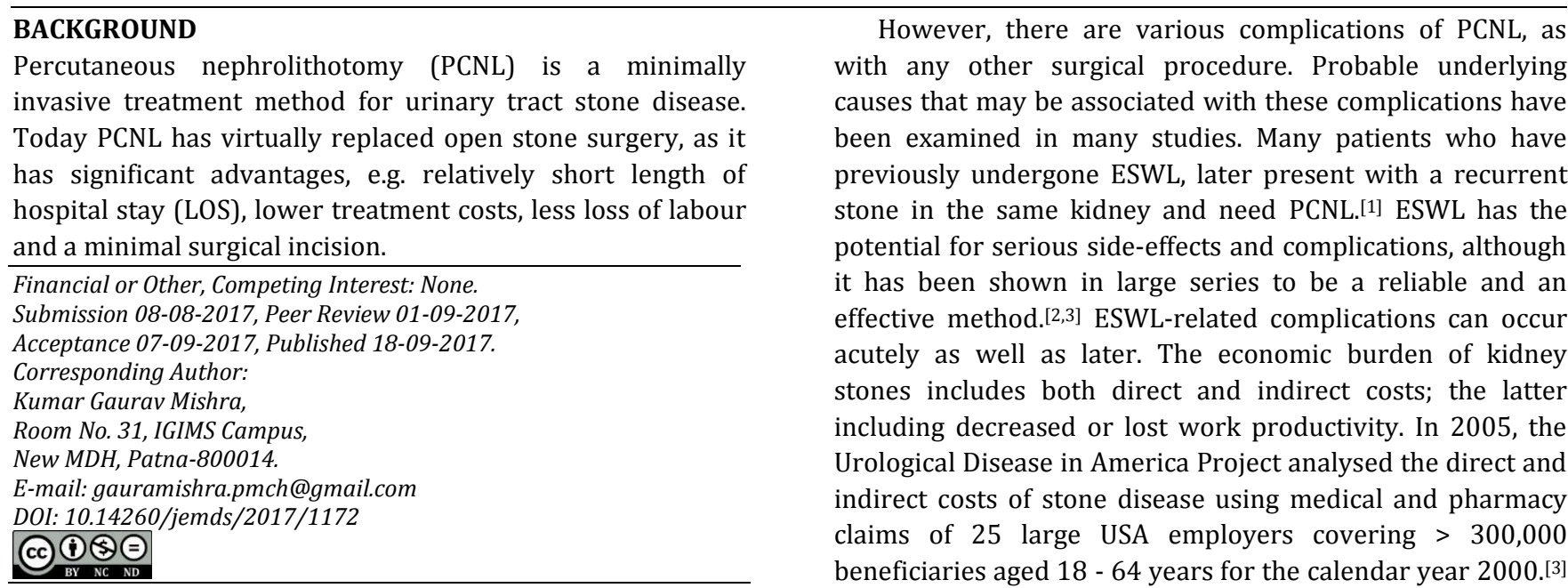


In the present study, we evaluated the effects of previous unsuccessful ESWL treatments (failed disintegration of stones or failed clearance of stones, not recurrences) on the performance and outcome of PCNL.

\section{MATERIALS AND METHODS}

This comparative study of the PCNL procedures performed in our Urology Department at Indira Gandhi Institute of Medical Sciences, Patna, between January 2015 and December 2016, 115 patients had similar stone burdens (cumulative stone burden $>600 \mathrm{~mm} 2$ ) as well as number of accesses and were included in this retrospective study. Stone size was evaluated by CT. Patients were divided into two groups: Group 1, comprised of 42 (36.5\%) patients, who underwent ESWL (failed disintegration of stones or failed clearance of stones) within 1 year prior to PCNL (to standardise all patients); Group 2 comprised of 73 (63.4\%) patients who had no ESWL. Haemoglobin $(\mathrm{Hb})$ levels and stone-free rates before and after PCNL were evaluated and compared. The demographic details of the groups are shown in Table 1. Complete blood count, serum creatinine, sodium, potassium, liver function tests, urine analysis, urine culture and antibiogram, and coagulation tests were performed preoperatively for each patient. In all patients a complete blood count was repeated 2 hrs. after PCNL. Anti-aggregant or anticoagulant treatments were discontinued for 7 days before PCNL. We excluded patients that had a bleeding tendency or abnormal coagulogram.

All patients were evaluated by CT preoperatively. All procedures were conducted according to the regulations of the Local Ethics Committee. Patients with a cumulative stone burden (for multiple stones, total area) of $>600 \mathrm{~mm} 2$, multiple accesses and incomplete data were excluded from the study.

\begin{tabular}{|c|c|c|c|}
\hline Variable & Group 1 & Group 2 & P value \\
\hline Number of patients (\%) & $42(36.5 \%)$ & $73(63.4 \%)$ & \\
\hline Age, years, mean & 45.6 & 46.1 & .98 \\
\hline BMI, kg/m2, mean & 28.1 & 27.1 & .87 \\
\hline Male/Female, $\mathrm{n}$ & $24 / 18$ & $41 / 32$ & .13 \\
\hline Stone Size, mm2, mean & 425 & 460 & .078 \\
\hline Side, right/left, n & $19 / 23$ & $35 / 38$ & .69 \\
\hline \multicolumn{4}{|c|}{ Table 1 } \\
\hline
\end{tabular}

The PCNLs were performed under general anaesthesia. The patients were placed in lithotomy position and an openended 6-F ureteric catheter was placed using a 22-F cystoscope with the correct placement of the catheter into the renal collecting system confirmed by fluoroscopy.

The ureteric catheter was stabilised using silk ties on to the urethral 16-F Foley catheter to prevent displacement during turning of the patient from a supine to prone position. The patient was prone positioned and the anaesthetist supervised the head and neck. The renal collecting system was imaged using retrograde contrast medium diluted with saline (1:1). After the introduction of the 18-G needle under fluoroscopy, the safety guidewire was inserted through the needle into the renal collecting system (generally the lower calyx), preferably into the ureter and renal parenchymal dilatation was performed up to 30 , although not in all patients. The 'bulls-eye' and 'triangulation' techniques were used for puncture. Amplatz dilators are generally preferred, because they are considered to be safer and more effective.
For an intercostal approach, the anaesthetist ensured safe entry by means of expiration. In patients who developed perioperative haemorrhage, blood transfusion was given if needed after assessing the perioperative haematocrit results and haemodynamic assessment by the anaesthetist.

Blood transfusions were also given to patients whenever appropriate according to their postoperative complete blood count. During the operation, the guidewire was introduced through the ureteric catheter and then removed via the ureteric catheter. When the operation was completed, a reentry malecot catheter was usually placed in such a way that it fitted into the renal pelvis. For those patients with haemorrhage, the nephrostomy tube was maintained, clamped until they were transferred to a bed. Generally, for all the patients the urinary catheter was removed on the first postoperative day. The nephrostomy tube of stable patients without haematuria was also removed on the first day. A ureteric stent was inserted in patients with long-term leakage, i.e. at 2 days after PCNL. Patient's demographics, stone characteristics, operative findings, success rate, need for auxiliary treatments and complications were documented in detail and compared in each group. Data were expressed as mean and standard deviation (SD). The Statistical Package for the Social Sciences for Windows (SPSS version 20.0; IBM Corporation, NY, USA) was used for statistical analysis. Numbers, means, percentages and intervals were assessed. Numbers and percentages were compared using the chisquare test. Before the comparison of mean values, the values were evaluated for homogeneity.

Homogenously distributed values were compared using the student's t-test and heterogeneously distributed values were compared using the Mann-Whitney U-test. Additionally, unilabiate and multivariate analyses were used to compare the effect of ESWL history and PCNL outcomes. A P $<0.05$ was considered to indicate statistical significance.

\section{RESULTS}

The mean (SD) age of the patients was 45.9 (14.1) years. Of the 115 patients included in the study, 50 (44\%) were women and 65 (56\%) were men. The mean (range) patient age in Group 1 was 45.6 (2 - 67) years and was 46.1 (19-66) years in Group 2. The mean (range) stone burden was 425 (220 - 440) $\mathrm{mm} 2$ in Group 1 and 460 (230 - 445) $\mathrm{mm} 2$ in Group 2. There were no differences between the groups for age, sex, weight and stone laterality. Table 1 lists the patient demographics and stone characteristics. For all the patients included in the study, intervention was achieved by a single access. Residual stones were identified in 28 (24.4\%) patients in the postoperative period. The stone-free rate was $74.8 \%$ (32 patients) in Group 1 and 76\% (56 patients) in Group 2.

Blood transfusion was needed in $5(4 \%)$ patients. These patients had a mean (range) body mass index (BMI) of 27.5 (25 - 31) kg/m2. The mean (range) amount of blood given to patients undergoing blood transfusion was 1.68 (1 - 4) units. At the end of the PCNL, nephrostomy tube was used in 107 (93\%) patients. The mean (range) time to nephrostomy tube withdrawal was $2.88(1-5)$ days and the mean (range) LOS was 3 (1 - 17) days. In Group 1 (ESWL-treated patients), residual stones were identified in $11(25.1 \%)$ patients and 8 units of blood were transfused in $5(7.6 \%)$. These patients had a mean (range) BMI of $27.1(26-30.3) \mathrm{kg} / \mathrm{m} 2$. The mean (range) LOS of ESWL treated patients was 3 (1 - 12) days and 
the mean (SD) time to nephrostomy tube removal was 2.8 (1.4) days. Of patients who had had no ESWL (Group 2), residual stones were identified in 18 (24\%) and 4 units of blood were transfused in $2(2 \%)$. The mean (range) LOS stay was $3(1-17)$ days and the mean (SD) time to nephrostomy tube withdrawal was $2.8(0.8)$ days. There was no statistically significant differences between the groups for LOS, nephrostomy tube removal time and the presence of residual stones $(\mathrm{P}=0.957, \mathrm{P}=0.961, \mathrm{P}=0.51$, respectively). The mean (SD, range) Hb level decrease in Group 1 was 3.4 (2.8, 0.2 6.1) g/dL and in Group 2 was 1.1 (3.2, $0.1-5.1) \mathrm{g} / \mathrm{dL}(\mathrm{P}=$ $0.01)$. In all $5(7.6 \%)$ patients in Group 1 and 2 (2\%) in Group 2 needed blood transfusions with most patients receiving 1 unit of blood. When both groups were evaluated for preoperative and postoperative $\mathrm{Hb}$ drop and the rate of blood transfusion, these were significantly more common in patients that had had ESWL treatment (both $\mathrm{P}=0.01$ ). There was no difference between the groups for either intraoperative or postoperative major and minor complications. There were no deaths.

Most of the complications were pain, bleeding, urine leakage after removal of the nephrostomy tube and postoperative fever. Pleural effusion as a major complication was seen in one patient in Group 1 and one patient in Group 2. They were treated conservatively.

Postoperative major infections such as pyelonephritis or sepsis developed in two patients in Group 1 and four patients in Group 2. A regional cellulitis developed on the needle access area in three patients in Group 2 and second generation cephalosporin was administered. Septic shock, damage to neighbouring organs and bowel perforation did not occur in any of our patients.

\section{DISCUSSION}

Urinary tract stone disease constitutes a substantial part of daily urological practice. The rate of developing kidney stones in one's lifetime has been reported to be $10 \% .{ }^{[5]}$ Current treatment options for kidney stones include ESWL, PCNL, ureteroscopy (URS), open surgery and laparoscopy. PCNL is a minimally invasive surgical method and today it has almost replaced open surgery. PCNL has significant advantages, e.g. short LOS, lower treatment costs, less labour loss. However, it has various complications as well just as any other surgical procedure. Many studies have evaluated various cases associated with these complications. The objective of the present study was to evaluate the efficacy and safety of PCNL in patients with a history of ESWL by comparing them with primary PCNL patients. In one of the first series on this subject published in 1985, Segura et al[6] examined 1000 PCNL cases and reported a major complication rate of $3.2 \%$. In that study, intraoperative bleeding was the most common complication occurring in six $(0.6 \%)$ patients and resulted in termination of the operation as necessary. In addition, six $(0.6 \%)$ patients who developed arterial venous fistula had embolisation and one patient underwent nephrectomy due to excessive postoperative bleeding, but no deaths were reported. In their study conducted in 1987, Lee et al[7] classified complications due to PCNL as major (Death, bleeding requiring surgical intervention, sepsis, urinary tract injury and neighbouring organs injury) or minor (Postoperative fever, bleeding requiring transfusion, extravasation, dislocation of nephrostomy, pneumonia and long-term wetting). Bleeding is one of the most significant complications of percutaneous entry of the upper urinary tract. The reported blood transfusion rate for percutaneous nephrostomy procedures is $0.5 \%-4 \% \cdot{ }^{[8]}$ In addition, the need for blood transfusion can rise from $6 \%$ up to $20 \%$ when opening the percutaneous tract with wider calibrations and with intrarenal manipulations during PCNL.[9,10] The factors associated with bleeding include: multiple entries, supracostal entry, dilatation of the tract, tract dilatation with different procedures other than with balloon dilatation, prolonged operation time and renal pelvis perforation.[8] However, most bleeds are from the renal parenchyma, which are not imperative in many cases. In cases of increased stone burden, especially staghorn stones, increased bleeding is likely during PCNL. Factors that may cause severe bleeding such as arteriovenous fistula or pseudoaneurysm are seen in $<0.5 \%$ of PCNL cases.[11]

Bleeding that requires treatment can occur in $1 \%$ of percutaneous operations.[12,13] The effect of ESWL on bleeding during PCNL is controversial. Many medical centres are concerned about the adverse effects of ESWL on tissues. ${ }^{[14]}$ Clinical and experimental studies have shown that this treatment might have severe, acute and chronic effects on the kidney and its peripheral tissues.[15-17] Acute renal changes after ESWL emerge as a significant decrease in effective renal plasma flow and GFR in the first $24-48$ h.[18] Possible chronic changes include an increase in blood pressure, decrease in renal function and increased recurrence of developing kidney stone. Renal damage in ESWL is known to be primarily avascular lesion. Acute and chronic renal disorders may appear after ESWL due to the development of severe damage at the microvascular level and at the interstitium in nephrons.[19,20] Renal parenchymal damage due to ESWL has been detected by MRI in $85 \%$ of patients.[21,22] Parenchymal damage may range from a small parenchymal lesion to the development of manifest haematoma.

Theoretically, these lesions may cause arterial hypertension as they do in renal trauma cases. The effects of lithotripsy on the kidney have been tested in animal models. Although, the primary studies conducted by Chaussy et al[23] showed a lack of pathological variations in dog's kidneys after lithotripsy, subsequent research showed that pathological changes may develop.[24] Thin-wall vessels are sensitive to shockwave damage. The haemorrhage extent is directly related to the kilovoltage used and number of shocks of the prescribed wave. ${ }^{[25]}$ Some studies have referred to the effects of lithotripsy on renal tubules and glomerulus cells. The appearance of detrimental effects after intense ESWL applications has been confirmed in many studies.[26,27] Stoller et al[28] retrospectively reviewed their cases and showed that previous ESWL history had no effect on bleeding in subsequent PCNLs.

However, brittle tissue and white membranes in damaged calyxes and the pelvicalyceal system were observed in another study, as the results of nephrostomy of the PCNL attempt following a failed ESWL.[29] They additionally expressed that fragmented and scattered stones in the calyx were likely to indicate an additional percutaneous access. However, the rates of success and complications were found to be similar in both patient groups, those who had undergone ESWL and those who had not. In the study by 
Resorlu et al[30] in 2010, the effect of PCNL on bleeding after open surgery and ESWL were evaluated, but no statistically significant difference was found between the groups. Mungan et al[31] evaluated patients who underwent PCNL after ESWL and found no significant statistical difference between the groups for residual kidney stones and blood transfusions. Although, success appears to be affected by the scar tissue that is likely to develop, we think that thickening of scar tissue and Gerota's fascia can be successfully overcome by Amplatz dilators. High success and low blood transfusion rates can be achieved by the effective use of imaging systems, the quality of the instruments used, developing technology and increasing surgical experience. In our present study, unlike other published studies, PCNL after ESWL treatment was found to increase the amount of bleeding and the need for blood transfusion. We enrolled patients who had stone burdens of $<600 \mathrm{~mm} 2$ and accessed with one attempt. Therefore, we think that our ESWL-treated patients more accurately represent the potential for blood loss during PCNL.

Although, fragmentation and easy collection of stones after ESWL has played a role in the success of the procedure, in the longer term it has been found that the fragmented stones tend to get stuck in the collecting system due to scar formation and hence traction applied to the kidney as well as the difficulty of the procedure can cause bleeding and this may lead to residual stones being left behind. Searching for a large number of broken stone pieces as a result of ESWL can extend the operation time, hence may lead to variations in bleeding and residual rates.[31] In the present study, ESWL treatment was shown not to affect the stone-free rates of patients with similar kidney stone burdens. In addition the other variables that we examined such as operation time, incidence of postoperative complications and LOS were shown not to be affected by ESWL pre-treatment as well. As for the limitations of our present study, we should mention that it had small number of patients enrolled and it was a retrospective study. However, unlike other published studies showing that ESWL has no effect on bleeding during PCNL, our present study has shown that ESWL increases perioperative bleeding and the need for transfusion. So our present study will incite reconsideration of the debate on this issue. Therefore, this point should be taken into consideration when examining our present data. Large scale studies are needed to evaluate the earlier effects of the ESWL and its effects on operational success.

\section{CONCLUSION}

Many patients who undergo PCNL have undergone ESWL treatment beforehand. The renal side-effects of ESWL are well known, the duration of which varies between studies. In the present study, we found that previous ESWL had an effect on bleeding during the PCNL procedure. The PCNL procedure can be applied with similar success rates after ESWL, but close attention should be paid to bleeding.

\section{Abbreviations}

$\mathrm{Hb}$ - Haemoglobin.

LOS - Length of hospital stay.

PCNL - Percutaneous nephrolithotomy.

\section{REFERENCES}

[1] Lojanapiwat B. Previous open nephrolithotomy: does it affect percutaneous nephrolithotomy techniques and outcome? J Endourol 2006;20(1):17-20.

[2] Bedir S, Kilciler M, Cincik M, et al. Relationship between extracorporeal shock wave lithotripsy and semen parameters in patients with lower ureteral stones. Fertil Steril 2004;82:1687-8.

[3] Hyams ES, Matlaga BR. Economic impact of urinary stones. Transl Androl Urol 2014;3(3):278-83.

[4] Tiselius HG, Ackermann D, Alken P, et al. Guidelines on urolithiasis $2007 . \quad$ Available at:https://www.guidelinecentral.com/summaries/gui delines-on-urolithiasis/\#h2_scope.

[5] Menon M, Resnick MI. Urinary lithiasis: etiology, diagnosis, and medical management. In: Walsh PC, Retik AB, Vaughan ED, et al. (eds). Campbell's urology. $8^{\text {th }}$ edn. Philadelphia, PA: WB Saunders Co 1988:3229305.

[6] Segura JW, Patterson DE, LeRoy AJ, et al. Percutaneous removal of kidney stones: review of 1,000 cases. J Urol 1985;134(6):1077-81.

[7] Lee WJ, Smith AD, Cubelli V, et al. Complications of percutaneous nephrolithotomy. AJR Am J Roentgenol 1987;148(1):177-80.

[8] Wolf JS. Percutaneous approaches to the upper urinary tract collecting system. In: Wein AJ, Kavoussi LR, Campbell MF, et al. (eds). Campbell-Walsh urology. $10^{\text {th }}$ edn. Philadelphia, PA: Elsevier Saunders 2012:P 1324.

[9] Havel D, Saussine C, Fath C, et al. Single stones of the lower pole of the kidney. Eur Urol 1998;33:396-400.

[10] McDougall EM, Liatsikos EN, Dinlenc CZ, et al. Percutaneous approaches to the upper urinary tract. In: Walsh PC, Retik AB, Vaughan ED, (eds). Campbells urology. $8^{\text {th }}$ edn. Philadelphia, PA: WB Saunders Co 2002:3320-60.

[11] Segura JW, Preminger GM, Assimos DG, et al. Nephrolithiasis clinical guidelines panel summary report on the management of staghorn calculi. The American urological association nephrolithiasis clinical guidelines panel. J Urol 1994;151(6):1648-51.

[12] Radecka E, Magnusson A. Complications associated with percutaneous nephrostomies. A retrospective study. Acta Radiol 2004;45(2):184-8.

[13] Kessaris DN, Bellman GC, Pardalidis NP, et al. Management of hemorrhage after percutaneous renal surgery. J Urol 1995;153(3 Pt 1):604-8.

[14] Tailly GG. Extracorporeal shock wave lithotripsy today. Indian J Urol 2013;29(3):200-7.

[15] Delius M, Enders G, Xuan ZR, et al. Biological effects of shock waves: kidney damage by shock waves in dogsdose dependence. Ultrasound Med Biol 1988;14(2):117-22.

[16] Knapp PM, Kulb TB, Lingeman JE, et al. Extracorporeal shock wave lithotripsy-induce perirenal hematomas. J Urol 1988;139(4):700-3.

[17] Lingeman JE, McAteer JA, Kempson SA, et al. Bioeffects of extracorporeal shock waves lithotripsy. J Endourol 1987;1:89-98.

[18] Thadhani R, Pascual M, Bonventre JV. Acute renal failure. N Engl J Med 1996;334:1448-60. 
[19] Calvino J, Romero R, Blanco $M$, et al. Cortical nephrocalcinosis induced by extracorporeal shock wave lithotripsy. Nephron 1999;81(2):242-3.

[20] Zhu Z, Xi Q, Wang S, et al. Percutaneous nephrolithotomy for proximal ureteral calculi with severe hydronephrosis: assessment of different lithotriptors. J Endourol 2010;24(2):201-5.

[21] Reis LO, Zani EL, Ikari 0, et al. Extracorporeal lithotripsy in children-the efficacy and long-term evaluation of renal parenchyma damage by DMSA99mTc scintigraphy. Actas Urol Esp 2010;34(1):7881.

[22] Iro H, Zenk J, Koch M. Modern concepts for the diagnosis and therapy of sialolithiasis. HNO 2010;58(3):211-7.

[23] Chaussy C, Brendel W, Schmiedt E. Extracorporeally induced destruction of kidney stones by shock waves. Lancet 1980;2(8207):1265-8.

[24] Hoscan MB, Ekinci M, Tunckiran A, et al. Management of symptomatic ureteral calculi complicating pregnancy. Urology 2012;80(5):1011-4.

[25] Penbegul N, Tepeler A, Sancaktutar AA, et al. Safety and efficacy of ultrasound-guided percutaneous nephrolithotomy for treatment of urinary stone disease in children. Urology 2012;79(5):1015-9.
[26] Karalezli G, Gogus O, Beduk Y, et al. Histopathological effects of extracorporeal shock wave lithotripsy on rabbit kidney. Urol Res 1993;21(1):67-70.

[27] Newman R, Hackett R, Senior D, et al. Pathologic effects of ESWL on canine renal tissue. Urology 1987;29(2):194-200.

[28] Stoller ML, Wolf JS, St Lezin MA. Estimated blood loss and transfusion rates associated with percutaneous nephrolithotomy. J Urol 1994;152(6 Pt 1):1977-81.

[29] Yuruk E, Tefekli A, Sari E, et al. Does previous extracorporeal shock wave lithotripsy affect the performance and outcome of percutaneous nephrolithotomy? J Urol 2009;181(2):663-7.

[30] Resorlu B, Kara C, Senocak C, et al. Effect of previous open renal surgery and failed extracorporeal shockwave lithotripsy on the performance and outcomes of percutaneous nephrolithotomy. J Endourol 2010;24(1):13-6.

[31] Mungan NA, Simsek AR, Tok A, et al. The effects of time period after open stone surgery and ESWL to the success rate of percutaneous nephrolithotomy. Turkiye Klinikleri J Urol 2014;5:39-45. 